
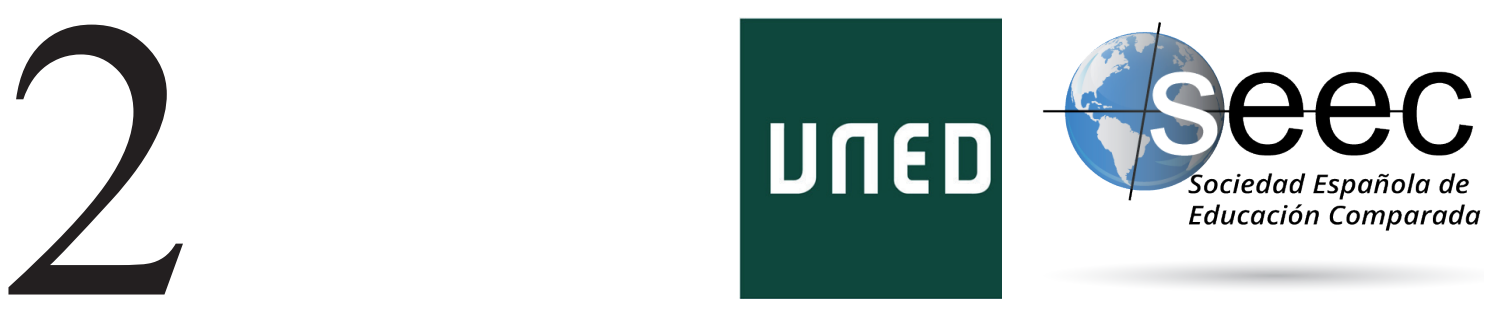

\title{
El acceso a la formación docente inicial en Europa: políticas e investigación
}

\author{
Access to initial teacher training in \\ Europe: policies and research
}

\section{Inmaculada Egido Gálvez*}

DOI: $10.5944 /$ reec.35.2020.24192

\author{
Recibido: 8 de abril de 2019 \\ Aceptado: 2 de octubre de 2019
}

\footnotetext{
*Inmaculada Egido Gálvez: es Doctora en Ciencias de la Educación por la Universidad Nacional de Educación a Distancia. Es Catedrática de Educación Comparada en la Facultad de Educación de la Universidad Complutense de Madrid y actualmente desempeña el puesto de Past President en la Junta Directiva de la Sociedad Española de Educación Comparada (SEEC). ORCID: http://orcid.org/oooo-00o3-1995-2688. Datos de contacto: E-mail: miegido@ucm.es
} 


\title{
Resumen
}

En los últimos años, el número de sistemas educativos europeos que han establecido procedimientos de selección previos a la entrada a las instituciones de formación inicial docente no ha dejado de incrementarse, siguiendo las recomendaciones de Organismos Internacionales como la OCDE y la Unión Europea. A partir de una revisión documental, este artículo presenta los sistemas de acceso a las instituciones de formación docente vigentes actualmente en Europa y sintetiza los resultados de los principales estudios de ámbito nacional e internacional que han profundizado en esta cuestión. La revisión muestra la existencia de tres grandes modelos de acceso a los estudios de profesorado en Europa y pone de manifiesto cómo el atractivo de la profesión docente en cada país condiciona los resultados de dichos sistemas de acceso. La conclusión es que los estudios de alcance internacional resultan indispensables para el análisis de esta temática, ya que aportan variabilidad con relación a las condiciones laborales de la docencia, como los salarios o el prestigio social, que no pueden ser captadas en los estudios de ámbito nacional. No obstante, se destaca la necesidad de profundizar en esta cuestión a través de la realización de investigaciones propiamente comparativas, que permitan avanzar más allá de los resultados aportados por las evaluaciones internacionales de rendimiento educativo. Dichas investigaciones comparadas harían posible considerar las diferentes realidades socioeducativas de cada contexto para analizar e interpretar modelos concretos de acceso a la formación. Asimismo, podrían contribuir a superar los mensajes simplistas de muchas de las recomendaciones sobre políticas de selección a la formación docente que se orientan a la imitación de los sistemas educativos de mejores resultados.

Palabras clave: Formación de profesores; Condiciones de admisión; Formación inicial, Europa; Política de la educación; Investigación comparativa

\begin{abstract}
In recent years, the number of European education systems that have established selection procedures prior to entry into initial teacher training institutions has continued to increase, following the recommendations of international organizations, such as the OECD and the European Union. Through a document review, this paper presents the systems of access to teacher training institutions in force in Europe and synthesizes the results of the main national and international studies that have deepened this issue. The review shows the existence of three major models of access to teacher training in Europe and point at how the attractiveness of the teaching profession in each country conditions the results of such access systems. The conclusion is that studies of international scope are essential for the analysis of this topic, since they provide variability in relation to the working conditions of teaching, such as salaries or social prestige, which cannot be captured in national studies. However, it is necessary to deepen in this issue through authentic comparative research, which allows us to advance beyond the results provided by the international large-scale educational assessments. Those comparative research would make it possible to consider the different socio-educational realities of each context to analyse and interpret concrete models of access to teacher training. Likewise, they could contribute to overcoming the simplistic messages of many of the recommendations on teacher selection policies aimed at imitating the best-performing education systems.
\end{abstract}

Keywords: Teacher education; Admission requirements; Initial training; Europe; Educational policy; Comparative research 


\section{El acceso a las instituciones de formación docente: planteamientos generales}

Desde hace algún tiempo, la investigación ha proporcionado una abundante evidencia empírica sobre la influencia del profesorado en los resultados académicos de los estudiantes. Aunque no es sencillo cuantificar el efecto de los docentes, se ha estimado que el profesorado explica aproximadamente el $30 \%$ de la varianza del rendimiento de los alumnos (Hattie, 2008). Algunos estudios afirman que la diferencia entre un buen y un mal profesor puede llegar a ser de un año de aprendizaje para sus alumnos en un solo curso académico (Hanushek, 2011; Hanushek, Rivkin y Schiman, 2016). En la misma línea, hay expertos que indican que tener maestros de alta calidad durante la escuela primaria puede compensar, o incluso eliminar, la desventaja inicial de los alumnos con un bajo nivel socioeconómico de procedencia (Rivkin, Hanushek y Kain, 2005).

Este interés por la vinculación entre la calidad docente y los logros de los estudiantes ha recibido en los años pasados un fuerte impulso adicional con la explotación de los datos de las evaluaciones internacionales de rendimiento. Dichas evaluaciones, sin abordar directamente la temática del profesorado, han hecho volver la vista hacia los modelos de profesión docente de los países que obtienen mejores resultados. Por ejemplo, el Programa para la Evaluación Internacional de los Alumnos (PISA) ha contribuido a que se multipliquen de manera exponencial los análisis de los factores vinculados al profesorado en países como Finlandia, Canadá o Corea del Sur, considerados «modelos ejemplares». Tanto PISA como otros estudios internacionales han aportado nuevas fuentes de información sobre este tema, lo que ha conducido a que los análisis sobre el mismo se hayan intensificado en el momento actual.

Por otra parte, los Organismos Internacionales dedicados a la educación han situado al profesorado como cuestión central de sus agendas, por lo que han contribuido de manera sustancial al refuerzo de la investigación sobre el mismo y a la difusión de sus resultados en todo el mundo. También desde los gobiernos y Ministerios de Educación de un gran número de países se diseñan y ensayan políticas destinadas a mejorar la profesión docente.

En este contexto de creciente atención al profesorado, la necesidad de establecer sistemas eficaces de acceso a la formación inicial docente ha ido ganando terreno con el tiempo. La Organización para la Cooperación y el Desarrollo Económico (OCDE) señaló hace ya algunos años la necesidad de «mejorar la selección a la formación docente», para lo que propuso introducir procedimientos destinados a valorar si las personas que quieren ser profesores tienen el conocimiento, las motivaciones, las habilidades y las cualidades personales necesarias antes de iniciar la formación (OECD, 2005).

Este organismo ha seguido trabajando sobre esta cuestión a lo largo de la pasada década. De hecho, el proyecto de la OCDE denominado TALIS-ITP (Initial Teacher Preparation), cuyos resultados se harán públicos en 2019, tiene entre sus focos principales de análisis la selección de los candidatos más adecuados para los programas de formación docente (OECD, 2017a).

$\mathrm{Al}$ igual que la OCDE, la Unión Europea (UE) ha formulado recomendaciones al respecto de la selección previa a la formación inicial docente. Aunque por razones de espacio no es posible realizar un repaso a las mismas, en la Comunicación de la Comisión Europea Un nuevo concepto de educación del año 2012 se puso ya de manifiesto la importancia de 
introducir sistemas adecuados y coherentes para la selección del profesorado (Comisión Europea, 2012). Más recientemente, la Comunicación Desarrollo escolar y docencia excelente para un gran comienzo en la vida subraya la necesidad de mejorar los procesos de selección «para identificar a las personas más adecuadas para la docencia» (Comisión Europea, 2017).

Simplificando en cierta medida, la justificación que desde la literatura emanada de los Organismos Internacionales se realiza sobre las ventajas que pueden derivarse de la implantación de sistemas de selección específicos y previos a la entrada a las instituciones de formación se apoya fundamentalmente en tres argumentos (Egido, 2019). Concretamente, la selección puede ayudar a:

- Identificar a los candidatos con más opciones de convertirse en buenos docentes en el futuro, lo que repercutirá en la mejora del sistema educativo.

- Localizar a los estudiantes que tienen más posibilidades de terminar su carrera con éxito.

- Regular el número de estudiantes admitidos a los centros de formación docente y racionalizar dicha formación, proporcionando una preparación de mayor calidad a un número más reducido de estudiantes.

Además de estos tres motivos, se considera también que introducir sistemas de selección que hagan de la docencia unos estudios de acceso restringido puede ser positivo para revalorizar la imagen y el prestigio social de la profesión, convirtiéndola en una opción más deseable para los estudiantes. Así, un estudio del Parlamento Europeo afirma:

«[...] los requisitos de selección para ingresar a las instituciones de formación docente pueden asegurar que solo accedan los "mejores" estudiantes, mejorar la imagen de la profesión y evitar perspectivas de empleo negativas» (Bokdam, van den Ende y Broek 2014: 29).

Del mismo modo, Andreas Schleicher, director de educación de la OCDE, considera que es posible mejorar el estatus de la profesión «a través de un reclutamiento selectivo, que hace que los profesores sientan que van a entrar en una carrera demandada por profesionales expertos» (Schleicher, 2012: 55).

\section{Panorámica de los sistemas de acceso a la formación docente en Europa}

Como cuestión previa a la presentación de los sistemas de acceso a la formación inicial docente es necesario considerar que los modelos de formación de profesorado en Europa son muy variados. Dichos modelos no solo difieren entre países sino, en ocasiones, en el seno de un mismo país, por lo que existe una gran heterogeneidad de sistemas de acceso a los mismos, difícil de simplificar. Por ello, es preciso apuntar que la siguiente exposición hace referencia al profesorado que trabaja en la etapa de educación obligatoria (enseñanza primaria y secundaria inferior) y se ciñe a los sistemas de acceso a las instituciones en las que se lleva a cabo la formación docente, sea esta la fase central de la preparación de los profesores o una etapa posterior tras la realización de otros estudios (como sucede en el caso de los másteres). 
La información ha sido recopilada a partir de la revisión de los informes nacionales sobre los sistemas educativos de 29 países (los 28 de la UE y Noruega) que ofrece en línea la Red Eurydice y la consulta a diversas publicaciones de carácter comparativo e internacional (Bokdam, Van den Ende y Broek, 2014; Caena, 2014; Darmody y Smith, 2016; Eurydice, 2018; Karras y Wolhuter, 2015; 2018; Morris y Patterson, 2013; OECD, 2017b). El análisis de ese conjunto de información permite ofrecer una panorámica general sobre los sistemas de acceso a la formación docente inicial vigentes actualmente en Europa (Figura 1).

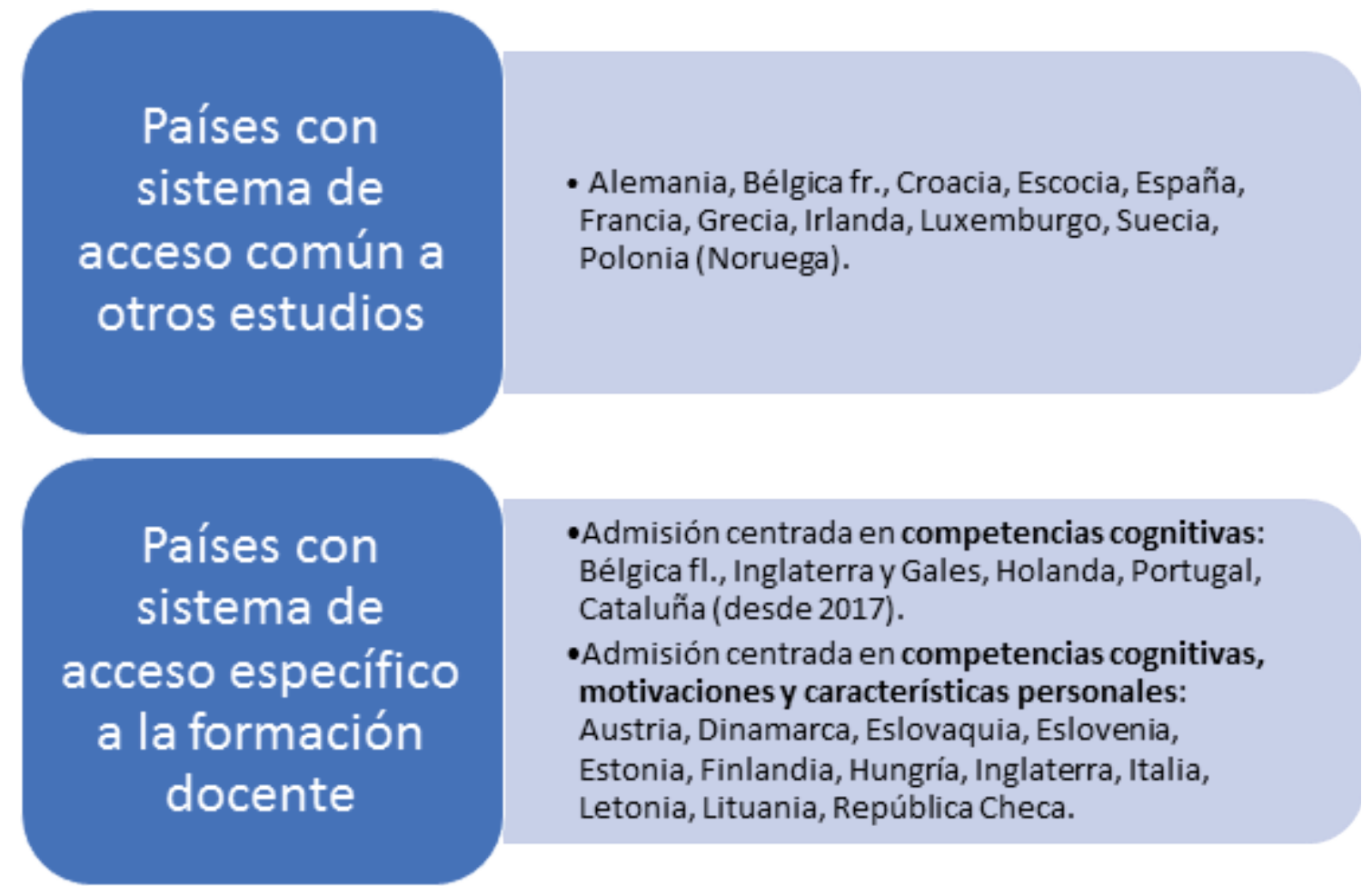

Figura 1. Modelos de acceso a la formación inicial docente en Europa. Fuente: Elaboración propia.

Como refleja la figura, los sistemas educativos europeos se dividen en dos grandes categorías. Los países con un sistema de admisión común a otros estudios son aquellos que establecen los mismos requisitos para acceder a la formación docente que para ingresar en otras carreras universitarias. Lo habitual es que tengan en cuenta las calificaciones de egreso de la secundaria, los resultados de las pruebas de acceso a la universidad o ambos. En el caso de que la formación docente tenga un nivel de postgrado, suelen utilizarse como criterio de acceso las calificaciones obtenidas en los grados. En varios de estos países se prioriza el rendimiento en matemáticas y lengua, así como habilidades y conocimientos específicos para los profesores de educación física, música o idioma. Este modelo es el vigente en Alemania, Bélgica francófona, Croacia, Escocia, Francia, Grecia, Irlanda, Polonia y Suecia, así como, fuera de la UE, Noruega. Es también el modelo utilizado en España, con excepción de Cataluña desde 2017 para los docentes de enseñanza primaria, dado que esta Comunidad introdujo a partir de ese año pruebas específicas de competencia comunicativa y razonamiento crítico y de competencia matemática para acceder a los estudios de Magisterio (Ametller y Codina, 2017). 
Aun cuando no existan requisitos específicos de acceso a las instituciones de formación docente inicial, en algunos países es habitual que las instituciones más demandadas establezcan sus propios criterios y mecanismos de selección, entre los que pueden incluirse entrevistas a los futuros candidatos (Eurydice, 2015).

Los países con un sistema de admisión específico para la formación docente, además de tener en cuenta lo contemplado en el modelo anterior, introducen requisitos específicos para el ingreso en las instituciones de formación docente. A su vez, en este grupo pueden diferenciarse dos modelos:

- Admisión centrada en los conocimientos, aptitudes o competencias cognitivas de los candidatos. Es el sistema vigente en Bélgica Flamenca, Inglaterra, Holanda, Portugal y, desde 2017, también Cataluña para el profesorado de primaria.

- Admisión centrada en las competencias cognitivas de los candidatos y en sus motivaciones y características personales en relación con la profesión docente. Es el procedimiento utilizado en Austria, Eslovaquia, Estonia, Finlandia, Hungría, Italia, Letonia, Lituania o la República Checa.

En el primer grupo lo habitual es que las aptitudes de los candidatos se evalúen por medio de pruebas escritas centradas en competencias numéricas y lingüísticas, aunque en algún caso, como el de Portugal, se contemplan solo estas últimas. En el segundo modelo, además de las pruebas de aptitud, se utilizan test psicológicos destinados a valorar las motivaciones y rasgos de personalidad de los aspirantes o bien se realizan pruebas orales y entrevistas. Los sistemas de selección más exigentes, como el de Finlandia, combinan distintos procedimientos, incluyendo exámenes escritos, pruebas orales y entrevistas (Pérez-Granados, 2015).

La responsabilidad de establecer el sistema de selección varía en función del país concreto que se analice. En unos casos, la definición de los procedimientos corresponde a la administración educativa central, en otros a la regional y en otros la administración educativa comparte con las instituciones de formación las responsabilidades al respecto, de modo que las dos instancias participan en la toma de decisiones (Eurydice, 2018).

El repaso a las fechas de implantación de sistemas específicos de selección para la formación docente muestra que muchos países los han establecido a lo largo de la pasada década. De hecho, el informe publicado por la red Eurydice en el año 2013 indicaba que un tercio de los países europeos contaba con estos sistemas de selección (Eurydice, 2013), mientras la situación en 2018 muestra que la cifra se ha incrementado a más de la mitad de los países.

\section{Aproximación a los resultados delos sistemas de acceso a la formación inicial docente}

Tras revisar los sistemas de acceso a la formación docente inicial, resulta de interés realizar una aproximación a los resultados que se obtienen con ellos. Con ese fin, es posible acudir a los estudios realizados en torno a ese tema en el ámbito de diferentes casos nacionales y a los trabajos que lo contemplan desde una perspectiva internacional. 


\subsection{Estudios de alcance nacional}

En el seno de los países europeos, las investigaciones rigurosas sobre los efectos de los sistemas de selección a la formación docente inicial son muy escasas, por lo que apenas es posible disponer de informaciones contrastadas. Prácticamente todas las evaluaciones de impacto que se han realizado sobre esta cuestión proceden de Estados Unidos y se han llevado a cabo desde enfoques econométricos, tratando de calcular los efectos derivados de la implantación de mecanismos de selección de los futuros docentes en el rendimiento académico de sus estudiantes (Vegas y Ganimian, 2013). Dichos estudios no arrojan resultados concluyentes, aunque tienden a mostrar que los profesores que obtienen altas calificaciones en la secundaria o en las pruebas de acceso a la universidad tienen alumnos con mejor rendimiento que los que obtuvieron calificaciones bajas. En todo caso, la relación encontrada es modesta (Hill-Jackson y Lewis, 2010; Cantrell et al., 2008).

También en el contexto de Estados Unidos algunas investigaciones apuntan a la existencia de lo que denominan «selección negativa» en los estudios de profesorado, indicando que los candidatos a la docencia tienen características cognitivas y de personalidad menos favorables que los candidatos a otros estudios. Ello no es solo problemático por el hecho de que personas poco cualificadas puedan ingresar en la profesión, sino también por el riesgo de que se desaliente de hacerlo a los candidatos mejor preparados (Vegas y Ganimian, 2013). Lo cierto es que incluso en el contexto norteamericano la evidencia sobre la selección negativa es contradictoria (Darling-Hammond, 2017) y en Europa no se corrobora en países como Alemania (Henoch, Klusmann, Lüdtke y Trautwein, 2015).

En el ámbito europeo, las informaciones disponibles a nivel nacional sobre los resultados de los sistemas de selección a la formación docente tienen un carácter básicamente descriptivo. Uno de esos resultados es que, como cabe esperar, cuando se introducen pruebas o requisitos específicos de acceso a las instituciones de formación inicial del profesorado se reducen tanto los candidatos potenciales como los admitidos a la misma. Este ha sido el caso de Holanda, donde tras la aplicación de las pruebas de acceso desde el año 2010 se han reducido los aspirantes a la formación docente en un 30 \%, afectando especialmente a los estudiantes procedentes de la Formación Profesional (Bokdam, van den Ende y Broek, 2014). Fuera de la Unión Europea, lo mismo ha sucedido en Noruega, que al incrementar las notas de entrada y exigir una puntuación determinada en lengua, matemáticas y ciencias sociales ha visto disminuido el número de candidatos en un 25 \% (Darmody y Smith, 2016). Algo más elevados son los datos de Cataluña, donde se estima que las pruebas de acceso han reducido el número de candidatos a Magisterio en un $40 \%$ (Vicens, 2018).

Por otra parte, se constata también que los países que solo valoran el rendimiento en la etapa de enseñanza secundaria, sin realizar pruebas específicas, no consiguen que los estudiantes que acceden a la formación hayan seleccionado la profesión docente en primera opción. De hecho, en sistemas como Grecia muchos de los estudiantes que entran a las instituciones de formación del profesorado no tienen la docencia como primera opción. La misma situación se producía en Holanda y Bélgica flamenca antes de introducir las pruebas de acceso y sucede también en países como España (Karras y Wolhuter, 2015; 2018). 


\subsection{Estudios de alcance internacional}

Complementando los estudios de ámbito nacional, los trabajos que cuentan con muestras internacionales pueden ser útiles para ayudar a comprender las variaciones que existen entre países (Park y Byun, 2015). Buena parte de estos trabajos se han realizado a partir de la información obtenida en las evaluaciones internacionales de rendimiento, tratando de poner en relación los procedimientos de selección del profesorado con los resultados obtenidos por los alumnos.

Comenzando con los datos de PISA, el conocido informe McKinsey de 2007 puso el foco de atención sobre este tema, al analizar las claves del éxito de los países que lideraron los resultados de la evaluación en 2006. En él se destacaba que uno de los rasgos compartidos por sistemas tan distintos como Canadá, Corea del Sur, Finlandia o Hong Kong era precisamente que estos países seleccionaban al profesorado entre los mejores estudiantes de secundaria. En ese informe aparecía una frase que a partir de entonces se ha repetido reiteradamente: «la calidad del sistema educativo no puede ser mejor que la de sus profesores» (Barber y Mourshed, 2007).

La misma consultora realizó en el año 2010 un estudio dedicado específicamente al tema de la selección del profesorado. Este trabajo volvió a subrayar la relación entre los resultados de los sistemas educativos y la selección de los futuros profesores, insistiendo en la idea de que los candidatos a la docencia deben reclutarse entre el tercio superior de los estudiantes de mejor rendimiento en la educación secundaria (lo que se denomina estrategia del tercio superior), siguiendo el ejemplo de Finlandia, Singapur y Corea del Sur (Auguste, Kihn y Miller, 2010).

Sin embargo, en la última edición de PISA, la de 2015, la OCDE se muestra mucho más cauta que McKinsey con relación a esta cuestión. Se constata que, efectivamente, cinco de los sistemas con mejores resultados (Finlandia, Hong-Kong, Macao, Taiwan y Singapur), tienen procesos muy exigentes y competitivos de selección a los programas de formación docente inicial, pero a la vez se pone de manifiesto que estos procesos existen también en países en los que los estudiantes tienen bajo rendimiento. Por otra parte, en otros sistemas de alto desempeño, como Japón, la selección docente se realiza al término de la formación. No hay, por tanto, un patrón claro que pueda identificarse (OECD, 2017b).

Por lo que se refiere a las evaluaciones desarrolladas en el marco de la Asociación Internacional para la Evaluación del Rendimiento Educativo (IEA), algunos trabajos han mostrado que los países que obtienen mejores resultados en el Estudio de Tendencias en Matemáticas y Ciencias (TIMSS) tienen a los mejores estudiantes como candidatos para acceder a la docencia (Sabrim, 2018). De igual modo, el estudio TEDS-M, destinado a analizar la formación inicial del profesorado de matemáticas, encontró que el rendimiento del profesorado en matemáticas durante sus estudios de secundaria era mayor en los países donde los resultados de los alumnos en TIMSS en esta materia son más altos. No obstante, en algunos países concretos no se pudo corroborar esta correlación (Tatto et al., 2012). Otros análisis realizados con los datos de TEDS-M apuntan también a que en los países que obtienen las mejores puntuaciones los procedimientos de selección del profesorado previos al acceso a la profesión son altamente exigentes (Ingvarson y Rowley, 2017).

Por último, pueden mencionarse los trabajos de Hanusek, Piopiunik y Wiederhold (2014) y Meroni, Vera-Toscano y Costa (2015), en los que se combinan los datos relativos 
al profesorado obtenidos en el Programa de Evaluación Internacional de Competencias de la Población Adulta de la OCDE (PIAAC) y los resultados de los alumnos en PISA, con el fin de averiguar el efecto de las competencias de los profesores en el rendimiento de los alumnos. Ambas líneas de investigación obtienen resultados que indican que las competencias de los docentes en lengua y matemáticas tienen un impacto significativo en el rendimiento de los estudiantes. La conclusión de Hanusek y sus colaboradores al respecto resulta contundente: «profesores más inteligentes producen alumnos más inteligentes» (Hanusek, Piopiunik y Wiederhold, 2014: 29).

En todo caso, y a pesar de afirmaciones como la mencionada, es importante tener en cuenta que los estudios revisados son correlacionales, por lo que realmente no puede hablarse en ellos de causa-efecto. Además, aunque en la literatura existe una tendencia general a encontrar relaciones positivas entre las habilidades cognitivas del profesorado y el rendimiento de los estudiantes, no siempre se corrobora dicha relación.

La investigación sobre la utilización de factores no cognitivos como criterios de selección para la formación docente, como la motivación de los candidatos o sus habilidades interpersonales, es aún muy escasa y con resultados poco concluyentes, aunque diversos estudios indican que los estudiantes que entran a la carrera con mayor motivación abandonan menos la profesión. El Estudio Internacional sobre Docencia y Aprendizaje (TALIS) de la OCDE aporta una medida indirecta de este tema, constatando que la motivación de los profesores hacia su profesión tiene una relación positiva con su percepción de autoeficacia (Egido, López-Martín, Manso y Valle, 2018). También algunos metaanálisis indican una relación, aunque modesta, entre las características de personalidad de los profesores y los logros de sus alumnos (Klassen y Tze, 2014).

\subsection{La importancia del contexto: el diferente atractivo de la profesión docente}

Es evidente que en un tema como el acceso a la formación docente el contexto de los distintos sistemas educativos puede condicionar, al menos en parte, los resultados de los distintos modelos empleados. Factores como la situación del mercado laboral en cada país o el atractivo de la profesión docente en el mismo resultan determinantes para entender la demanda de estudiantes interesados en ingresar en las instituciones de formación. Por ello, es necesario profundizar en los factores de atracción y expulsión a la docencia como opción profesional en cada contexto. Dichos factores se relacionan con motivaciones intrínsecas, como el deseo de trabajar con niños o de contribuir a mejorar la sociedad, así como con motivaciones extrínsecas, entre las que se encuentran las remuneraciones, el prestigio social de la profesión e incluso estereotipos de género, que hacen que los varones contemplen la docencia como posible ocupación laboral en menor medida que las mujeres (Ashby et al., 2008).

Algunos estudios internacionales han analizado este tipo de cuestiones, profundizando en las diferencias que existen entre países con relación al atractivo de la docencia como opción profesional. Por ejemplo, la OCDE ha realizado dos publicaciones al respecto, basadas en los datos de PISA 2006 y 2015, con el título ¿Quién quiere ser profesor? (OECD, 2015; 2018b). El primero de estos estudios indica que un $5 \%$ de los alumnos de 15 años en los países de la OCDE se veía a sí mismo como profesor en un futuro, con claras diferencias por género (3\% de chicos frente a $6 \%$ de chicas). Esta cifra se redujo ligeramente en PISA 2015, con un resultado del 4,4\%, persistiendo las diferencias de género (OECD, 2018a). Los resultados obtenidos en ambos estudios muestran que, en 
términos globales, las habilidades en lengua y matemáticas de los alumnos que quieren ser docentes tienden a ser más bajas que las de quienes aspiran a otras profesiones que requieren estudios universitarios en el conjunto de los países de la OCDE (OECD, 2015; 2018a). Sin embargo, un análisis detallado de los datos permite apreciar las diferencias que a este respecto se encuentran entre países. Así, mientras en Dinamarca, Finlandia, Japón o Suiza, por citar solo algunos ejemplos, no existen diferencias entre ambos grupos, en Austria, España, Holanda o la República Checa los estudiantes que se ven a sí mismos como docentes en el futuro tienen un rendimiento más bajo en matemáticas que los que optan por otras profesiones (OECD, 2015).

Además de lo anterior, el estudio muestra también que existe una correlación positiva entre el porcentaje de jóvenes que quiere ser docente en un país y los salarios del profesorado en relación con los de otros titulados universitarios en ese país (OECD, 2015; 2018a).

Por su parte, el trabajo de Park y Byum (2015) se centró específicamente en los alumnos de alto rendimiento (tercio superior) en la educación secundaria de 23 países de la OCDE, tratando de averiguar por qué algunos de estos países tienen éxito en atraer a los mejores estudiantes hacia la docencia y otros no. El análisis constata la relación positiva entre las remuneraciones relativas del profesorado y las expectativas de ser profesores entre estos alumnos. Pero, además, muestra también la relación que existe entre dichas expectativas y el estatus social de la profesión docente en cada país, medido a través del índice global de estatus del profesorado de la Fundación VarKey Gems. La correlación es significativa para los estudiantes varones, y, más aún, en el caso de las mujeres de alto rendimiento en secundaria.

En una línea similar, Dolton y Marcerano-Gutiérrez (2011) encontraron una relación clara de signo positivo entre salarios del profesorado y rendimiento de alumnos en varias ediciones de PISA y TIMSS. Su conclusión es que salarios más altos y aumentos salariales más rápidos parecen permitir el reclutamiento de personas de mayor capacidad.

Otros estudios de alcance internacional han puesto también de manifiesto esta relación, contradiciendo la tesis de que los factores determinantes para elegir la docencia como opción profesional son las motivaciones intrínsecas y no las extrínsecas. Entre ellos, puede mencionarse el informe elaborado para la Comisión Europea por Carlo et al. (2013), en el que los propios profesores en ejercicio indican que el atractivo de la profesión docente se encuentra vinculado a los salarios y al prestigio social. De igual modo Han, Borgonovi y Guerriero (2018) destacan que entre las motivaciones que impulsan a los estudiantes de secundaria a trabajar como docentes se encuentran los salarios, la consideración social sobre la importancia del trabajo de los profesores y el respeto a los mismos.

\section{Conclusiones}

La preocupación actual por el profesorado como factor esencial para la calidad educativa ha derivado en un creciente interés por los sistemas del acceso a las instituciones de formación docente inicial en el ámbito europeo. Los Organismos Internacionales formulan recomendaciones al respecto y cada vez más países establecen procedimientos específicos de selección previos al inicio de etapa de formación. Sin embargo, resulta difícil definir unos criterios que permitan identificar a los candidatos con el potencial de ser docentes competentes en el futuro, puesto que aún no se dispone de un conocimiento sólido que identifique las capacidades y habilidades que definen a un buen docente. Este hecho 
se refleja en la existencia de distintos sistemas de acceso vigentes en Europa en estos momentos. Algo menos de la mitad de los países tienen el mismo sistema para acceder a la formación docente que para otros estudios universitarios, utilizando como criterio general las calificaciones de la enseñanza secundaria y de las pruebas de acceso a la universidad. El resto de los sistemas europeos cuenta con procedimientos específicos de selección para las instituciones de formación docente, si bien mientras unos se centran exclusivamente en competencias de tipo cognitivo, otros valoran también factores como la motivación hacia la profesión, los rasgos de personalidad y las actitudes de los candidatos.

La investigación sobre los resultados obtenidos con esos distintos sistemas es aún muy incipiente. Es lógico suponer que los procedimientos que contemplan múltiples criterios de selección para el acceso a la formación docente son más adecuados que los que contemplan una única dimensión, pero lo cierto es que no disponemos de evidencias suficientes que corroboren esta idea y que pongan en relación los costes de dichos sistemas con los efectos de los mismos (Klassen y Kim, 2019).

Los estudios con muestras internacionales realizados a partir de las evaluaciones educativas a gran escala han comenzado a proporcionar nuevas pistas sobre este tema, superando algunas de las limitaciones de los análisis de ámbito nacional, que raramente pueden tener en cuenta la variabilidad de factores como las remuneraciones y el prestigio social de la enseñanza como ámbito laboral. Como cabe esperar, dichos estudios han puesto de manifiesto la importancia del atractivo profesional de la docencia en los distintos países. Los procedimientos de selección están muy vinculados a la existencia de una demanda suficiente de candidatos para los estudios, que a su vez guarda relación con los salarios y con la consideración de la profesión docente en cada país. La selección se dificulta en los países con escasez de demanda, donde el reto es atraer a un número suficiente de candidatos.

Por otra parte, la evidencia empírica aportada por los estudios internacionales sobre las relaciones entre la selección de los candidatos a la formación inicial, sus resultados durante y después de la formación y el rendimiento de sus estudiantes es poco concluyente aún, dado que resulta extremadamente complejo desentrañar los efectos de la selección de los debidos a otras variables. A pesar de ello, la mayor parte de dichos estudios parece apoyar el establecimiento de sistemas específicos de selección para el acceso a la formación del profesorado.

No cabe duda de que aún se necesita mucha investigación sobre este tema, destacando al respecto la importancia de realizar estudios propiamente comparativos, que pongan en relación contextos, sistemas de selección y resultados a partir del análisis en profundidad de diferentes modelos nacionales o regionales. Sin negar el valor de los trabajos basados en los datos de evaluaciones de rendimiento a gran escala, lo cierto es que estos también presentan limitaciones. Entre dichas limitaciones, resulta relevante la marginación del contexto y la ausencia de un análisis cultural en profundidad de los diferentes casos analizados. Junto a lo anterior, la mayoría de estos estudios contemplan los sistemas de acceso a la formación docente como una variable aislada, sin considerar las relaciones que esta tiene con el resto del sistema de formación y acceso a la docencia en cada país. Adicionalmente, las conclusiones de estos trabajos tienden a proporcionar mensajes simplistas con vistas a la implementación de políticas educativas, proponiendo recomendaciones basadas en la imitación de los modelos de los países de mayor éxito (Schleicher, 2016). 
Los estudios comparados sobre los sistemas de acceso a la formación docente permitirían superar en cierta medida algunas de las limitaciones de las evaluaciones a gran escala, tomando en consideración factores que las mismas no atienden. A este respecto, el análisis de la situación del mercado laboral en los distintos países y regiones tiene que ser contemplado, pues se trata de un factor claramente relevante para entender la demanda de la profesión docente en ellos. De igual modo, los estudios descriptivos precedentes a la comparación permitirían profundizar en las variaciones que pueden existir entre etapas educativas, especialidades y tipos de instituciones de formación dentro de cada uno de los sistemas analizados. Junto a lo anterior, la comparación en profundidad de diferentes casos facilitaría el análisis de las interrelaciones entre las políticas de profesorado en los distintos sistemas educativos (Vegas y Manimian, 2013), de manera que las recomendaciones derivadas de la misma con relación a los modelos de acceso pudieran formularse teniendo en cuenta el contexto en el que van a establecerse. Esta consideración del contexto, que es consustancial a la investigación comparativa, resulta esencial para diseñar políticas de acceso a la formación docente que resulten efectivas.

\section{Referencias bibliográficas}

Ametller, J. y Codina, F. (Coords.) (2017). La mejora de la formación inicial de docentes. Reflexiones y propuestas. Barcelona: Programa de mejora e innovación en la formación inicial de maestros. Colección Documentos MIF, 4. http:// www.educacionyfp.gob.es/yosoyprofe/dam/jcr:ogbbccec-94c8-4bed-9c4470740df54bf2/180629\%20modeloformativoes.pdf

Ashby, P., Hobson, A. J., Tracey, L., Malderez, A., Tomlinson, P. D., Roper, T., Chambers, G. N. y Healy, J. (2008). Beginner Teachers' Experiences of Initial Teacher Preparation, Induction and Early Professional Development: A Review of Literature. Nottingham: University of Nottingham.

Auguste, B. G., Kihn, P. y Miller, M. (2010). Closing the Talent Gap: Attracting and Retaining Top-third Graduates to Careers in Teaching: An International and Market Research-based Perspective. McKinsey. https://www.mckinsey.com/ / media/mckinsey/industries/social\%2osector/our\%2oinsights/closing\%20 the\%20teaching\%20talent\%20gap/closing-the-teaching-talent-gap.ashx

Barber, M. y Mourshed, M. (2007). How the World Best-performing School Systems Come out on Top. McKinsey y Company. http://www.mckinsey.com/locations/ ukireland/publications/pdf/Education_report.pdf

Bokdam, J., van den Ende, I. y Broek, S. (2014). Teaching Teachers: Primary Teacher Training in Europe. State of Affairs and Outlook. European Parliament Policy Department B: Structural and Cohesion Policy. http://www.europarl.europa.eu/ RegData/etudes/STUD/2014/529068/IPOL_STU(2014)529068_EN.pdf

Caena, F. (2014). Initial Teacher Education in Europe: an Overview of Policy Issues. European Commission. ET2020 Working Group on Schools Policy. http:// ec.europa.eu/dgs/education_culture/repository/education/policy/strategicframework/expert-groups/documents/initial-teacher-education_en.pdf 
Cantrell, S., Fullerton, J., Kane, T. J. y Staiger, D. O. (2008). National Board Certification and Teacher Effectiveness: Evidence from a Random Assignment Experiment. NBER Working Paper $\mathrm{n}^{0}$ 14.608. Cambridge, MA: National Bureau of Economic Research. DOI: 10.3386/w14608

Carlo, A., Michel, A., Chabanne, J. C., Bucheton, D., Demougin, P., Gordon, J., Sellier, M., Udave, J. P. y Valette, S. (2013). Study on Policy Measures to Improve the Attractiveness of the Teaching Profession in Europe. Research Report EAC-20101391. Luxembourg: Publications Office of the European Union, 2 vols.

Comisión Europea (2012). Comunicación de la Comisión al Parlamento Europeo, al Consejo, al Comité Económico y Social Europeo y al Comité de las Regiones "Un nuevo concepto de educación: invertir en las competencias para lograr mejores resultados socioeconómicos». COM/2012/0669 final.

Comisión Europea (2017). Comunicación de la Comisión al Parlamento Europeo, al Consejo, al Comité Económico y Social Europeo y al Comité de las Regiones "Desarrollo escolar y docencia excelente para un gran comienzo en la vida". $\mathrm{COM} / 2017 / 0248$ final.

Darling-Hammond, L. (2017). Teacher Education around the World: What can we Learn from International Practice? European Journal of Teacher Education, 4O(3), pp. 291-309. https://doi.org/10.1080/02619768.2017.1315399

Darmody, M. y Smyth, E. (2016). Entry to Programmes of Initial Teacher Education. Dublin: The Economic and Social Research Institute. https://www.esri.ie/pubs/ BKMNEXT299.pdf

Dolton, P. y Marcenaro-Gutierrez, O. D. (2011). If You Pay Peanuts Do You Get Monkeys? A Cross-country Analysis of Teacher Pay and Pupil Performance. Economic Policy, 26(65), pp. 5-55. https://doi.org/10.1111/j.1468-0327.2010.00257.x

Egido, I. (2019). Profesión docente y carrera docente, retos en el marco de la Unión Europea. En Consejo Escolar de la Comunidad de Madrid (Ed.): La mejora de la profesión docente en la Comunidad de Madrid (pp. 41-55). Madrid: Comunidad de Madrid. http://www.madrid.org/bvirtual/BVCM016442.pdf

Egido, I., López-Martín, E., Manso, J. y Valle, J. M. (2018). Determining factors of teachers' self-efficacy in countries of the European Union. Results from TALIS. Educación XX1, 21(2), 225-248. DOI: http://dx.doi.org/10.5944/educxx1.15875

Eurydice (2013). Cifras clave del profesorado y la dirección de centros educativos en Europa. Luxemburgo: Oficina de Publicaciones de la Unión Europea.

Eurydice (2015). The Teaching Profession in Europe: Practices, Perceptions, and Policies. Luxembourg: Publications Office of the European Union.

Eurydice (2018). Teaching Careers in Europe: Access, Progression and Support. Luxembourg: Publications Office of the European Union.

Han, S. W., Borgonovi, F., y Guerriero, S. (2018). What Motivates High School Students to Want to Be Teachers? The Role of Salary, Working Conditions, and Societal EvaluationsAboutOccupationsinaComparativePerspective.AmericanEducational Research Journal, 55(1), pp. 3-39. https://doi.org/10.3102/o002831217729875 
Hanushek, E. A. (2011). The Economic Value of Higher Teacher Quality. Economics of Education Review, 3o, pp. 466-479.

Hanushek, E. A., Piopiunik, M., y Wiederhold, S. (2014). The value of smarter teachers: International evidence on teacher cognitive skills and student performance (No. w20727). Cambridge: National Bureau of Economic Research. https://www.nber. org/papers/w20727

Hanushek, E. A., Rivkin, S. G. y Schiman, J. C. (2016). Dynamic effects of teacher turnover on the quality of instruction. Economics of Education Review, 55, pp. 132-148.

Hattie, J. (2008). Visible Learning: A Synthesis of Meta-analyses relating to Achievement. New York: Routledge.

Henoch, R. J., Klusmann, U., Lüdtke, O., y Trautwein, U. (2015). Who becomes a teacher? Challenging the "negative selection" hypothesis. Learning and Instruction, 36, pp. 46-56. DOI: 10.1016/j.learninstruc.2014.11.005

Hill-Jackson, V. y Lewis, C. (Eds.) (2010). Transforming Teacher Education: What Went Wrong with Teacher Training and How We Can Fix it. Sterling: Stylus.

Ingvarson. L. y Rowley, G. (2017). Quality Assurance in Teacher Education and Outcomes: A Study of 17 Countries. Educational Researcher, 46(4), pp. 177-193. https://doi. org/10.3102/0013189X17711900

Karras, K. G. y Wolhuter, C. C. (Eds.) (2015). International Handbook of Teacher Training and Re-training Systems in Modern World. Nicosia: Studies and Publishing.

Karras, K. G. y Wolhuter, C. C. (Eds.) (2018). International Handbook on Teacher Education Worldwide, 3 vols. Nicosia: Studies and Publishing.

Klassen, R. M. y Tze, V. M. C. (2014). Teachers Self-efficacy, Personality, and Teaching Effectiveness: A Meta-analysis. Educational Research Review, 12, pp. 59-76.

Klassen, R. M. y Kim, L. E. (2019). Selecting teachers and prospective teachers: A metaanalysis. Educational Research Review, 26, pp. 32-51.

Meroni, E. C, Vera-Toscano, E. y Costa, P. (2015). Can low skill teachers make good students? Empirical evidence from PIAAC and PISA. Journal of Policy Modeling, 37(2), pp. 308-323. https://doi.org/10.1016/j.jpolmod.2015.02.006.

Morris, J., y Patterson, R. (2013). Around the World: The Evolution of Teaching as a Profession. Wellington: New Zealand Initiative. https://nzinitiative.org.nz/ reports-and-media/reports/around-the-world/

OECD (2005). Teachers Matter. Attracting, Developing and Retaining Effective Teachers. Paris: OECD.

OECD (2015). Who wants to become a teacher? PISA in Focus 58. Paris: OECD. DOI: http://dx.doi.org/10.1787/22260919

OECD (2017a). TALIS Initial Teacher Preparation Study. Paris: OECD. http://www. oecd.org/education/school/talis-initial-teacher-preparation-study.htm\#About 
OECD (2017b). What do we know about teachers'selection and professional development in high-performing countries? PISA in Focus 70. Paris: OECD. http://dx.doi. org/10.1787/22260919

OECD (2018a). Effective Teacher Policies: Insights from PISA. Paris: OECD. https:// doi.org/10.1787/9789264301603-7-en

OECD (2018b), "Who wants to become a teacher and why?", Teaching in Focus, 22. Paris, OECD. https://doi.org/10.1787/d6a81156-en.

Park, H. y Byun, S. (2015). Why Some Countries Attract More High-Ability Young Students to Teaching: Cross-National Comparisons of Students' Expectation of Becoming a Teacher. Comparative Education Review, 59(3), pp. 523-549. doi: $10.1086 / 681930$

Pérez-Granados, L. (2015). La selectividad universitaria en España y Finlandia. Criterios, estrategias e instrumentos de selección de candidatos para la formación inicial de profesorado. Tesis Doctoral. Málaga: Universidad de Málaga. https:// riuma.uma.es/xmlui/handle/10630/10578

Rivkin, S. G., Hanushek, E. A. y Kain, J. F. (2005). Teachers, Schools and Academic Achievement. Econometrica, 73(2), pp. 417-458.

Sabrin, M. (2018). Elementary Teacher Education in the Top Performing European TIMSS Countries: A Comparative Study. International Education Studies, 11(4). https://doi.org/10.5539/ies.v11n4p152

Schleicher, A. (Ed.) (2012). Preparing Teachers and Developing School Leaders for the 21st Century: Lessons from around the World. Paris: OECD. http://dx.doi. org/10.1787/9789264xxxxxx-e

Schleicher, A. (2016). Teaching Excellence through Professional Learning and Policy Reform: Lessons from Around the World. International Summit on the Teaching Profession. Paris: OECD. http://dx.doi.org/10.1787/9789264252059-en

Tatto, M. T., Schwille, J., Senk, S. L., Ingvarson, L., Rowley, G., Peck, R., Bankov. K., Rodríguez, M. y Reckase, M. (2012). Policy, Practice, and Readiness to Teach Primary and Secondary Mathematics in 17 Countries: Findings from the IEA Teacher Education and Development Study in Mathematics (TEDS-M). Amsterdam: International Association for the Evaluation of Educational Achievement.

Vegas, E. y Ganimian, A. (2013). Teoría y evidencia sobre las políticas docentes en países desarrollados y en desarrollo. IDB Working Paper Series, No. IDBWP-438. http://repositorio.minedu.gob.pe/bitstream/handle/123456789/3370/ Teor\%C3\%ADa_y_evidencia_sobre_las_pol\%C3\%ADticas_docentes_en_ pa\%C3\%ADses_desarrollados_y_en_desarrollo.pdf?sequence $=1$

Vicens, L. (2018). El 40\% d'aspirants a mestre no superen les proves d'aptitud. Ara, 7 de julio de 2018. https://www.ara.cat/societat/aspirants-mestre-superen-provesaptitud_o_2047595269.html 